\title{
HUMAN-MACHINE COLLECTIVE INTELLIGENCE FOR DECISION SUPPORT: GENERAL VISION
}

\author{
Alexander Smirnov and Andrew Ponomarev \\ St. Petersburg Institute for Informatics and Automation of the Russian Academy of Sciences \\ 39, 14 th Line, 199178, St. Petersburg, Russian Federation
}

\begin{abstract}
Most of the existing approaches to organize collective work of humans and machines (e.g., in the scope of crowdsourcing or crowd computing) utilize pre-defined workflow specifications. Although strict limitation of the participant's capabilities pays back in a wide range of applications, creative and organizational abilities of a human in such systems are mostly discarded. Decision support systems, on the other hand, require flexible workflow, because decision making very often is based on interactive and iterative exploration of the problem. The paper discusses a novel class of decision support systems, that are based on an environment, leveraging human-machine collective intelligence, i.e. environment that supports humans and software services jointly working towards a common goal. The distinctive feature of the environment is support for natural self-organization processes in the community of participants (without a pre-defined workflow). The paper outlines general vision of the proposed environment and enumerates a set of foundational technologies and enablers.
\end{abstract}

\section{KEYWORDS}

Collective Intelligence, Artificial Intelligence, Human-Machine Systems, Human-in-the-Loop, Decision Support

\section{INTRODUCTION}

Current limitations of "pure" programmatic and artificial intelligence (AI) based solutions to many real-life problems has led to the emergence of crowd-based systems - information processing systems where some operations are done by humans (who participate in the system via Internet). In most crowd-based systems, workflow or algorithm is specified in design time. However, for many problems occurring in highly dynamic domains it is not viable to arrange a well-defined workflow in advance, because the situation may rapidly change. A natural technological answer to that is development of a new generation of human-machine systems, characterized by adaptiveness. First steps in the direction of creating computational systems including humans and giving them more freedom to define possible actions and a way of solving a problem has already been done ("flash organizations" (Retelny et al., 2017; Valentine et al., 2017), collective adaptive systems (CAS) (Viroli et al., 2018), hybrid CAS (Dustdar et al., 2017) etc.).

Decision-making is one of the types of problems that require flexible workflow, because decision-making very often is based on interactive and iterative exploration of the problem. Besides, modern decision-making utilizes variety of knowledge sources and information processing tools (software services). Therefore, it is important to create methods (and a set of technologies implementing them) that would allow collectives of people and software services with elements of AI to provide decision support defining the required activities in a flexible way.

The paper presents one of the steps in this direction, describing the general vision and a set of foundational principles for a decision support environment providing wide range of opportunities to support self-organization, paving the way to flexible and adaptive self-organizing communities of participants solving complex problems. 


\section{RELATED WORK}

The overwhelming part of research in the field of human-machine computing (crowdsourcing, crowd-computing) systems understands human participant as a special type of "computing device" that can process requests of a certain type. During the design time, the whole information processing workflow is build and operations requiring human processing are identified. In the run time the function of a human participant, is reduced to performing a specific task, proposed to him/her by the system, interacting with it in a strictly limited manner (Kulkarni et al., 2011; Ahmad et al., 2011; Barowy et al., 2012; Minder \& Bernstein, 2012; Tranquillini et al., 2015).

Although such a rigid division of roles (designer vs. participant of the system) and strict limitation of the participant's capabilities pay back in a wide range of tasks (usually simple ones like annotation, markup, etc.), the creative and organizational abilities of a person in such systems are discarded. The experimental systems where human participants could refine the workflow appeared in 2012 (Kulkarni et al., 2012), but the problem is starting to receive the closest attention of the research community only nowadays. In particular, in recent years several studies have appeared on the limitations of systems based on the hard flow of work (Retelny et al., 2017) and proposing the formation of dynamic organizations from members of the crowd community (the so-called flash organizations (Valentine et al., 2017)). These studies, however, do not explicitly use the existing body of knowledge on self-organization: descriptive (Dale et al., 2013; Kogan, 2016), formal (Berry, 1992), and constructive (Gorodetskii, 2012). On the other hand, none of the existing studies of self-organization processes provide a set of enactable mechanisms, suitable for support of self-organization in human-machine collectives (where both humans and machines can play active role).

\section{VISION OF THE DECISION SUPPORT ENVIRONMENT}

The proposed environment aims at supporting the process of making complex decisions and/or making decisions in complex problem domains. The complexity of making such decisions generally stems from problem uncertainty in many levels and the lack of relevant data at decision maker's disposal. Therefore, while in the upper level the methodology of decision-making stays quite definite (identification of the alternatives, identification of the criteria, evaluation of the alternatives etc.), the exact steps required to collect all the needed data, analyze it and present to the decision maker may be unclear. That is why decision support requires ad hoc planning of the low-level activities and should leverage self-organizing capabilities of the participants of the decision support process. Besides, currently most of the complex decisions are based not only on human intuition or expertise, but also on the problem-relevant data of various types and sources (starting from IoT-generated, to high-level Linked Data), processed in different ways. In other words, decision support is in fact human-machine activity, and the environment just offers a set mechanisms and tools to mitigate this activity.

The problem of supporting complex decisions touches the interests of the following roles. First, decision-makers, who need an access to the relevant expertise (not only in terms of domain knowledge, but also operational - what should be done to estimate absent parameters). Second, experts, who can provide this expertise, and, under some remuneration model may even be motivated to do so. Third, data and service providers, who are motivated to provide some problem-specific data and tools in exchange for payments.

The problem of making such decisions is ubiquitous and its impact spreads to virtually all areas of human activities, especially in complex domains: smart city, business management, environment problems, government and so on.

Successful solution would be an environment where participants of different nature (human and machine) could be able to communicate and decide on the particular steps of decision support process, perform these steps and exchange results, motivated by some external or internal mechanisms, making the whole environment profitable for all parties.

As it was already mentioned, there are three main categories of users: decision-makers, experts, service providers.

Decision-makers are responsible for the analysis of a situation and making a decision. In some cases, where the uncertainty associated with the situation is too high, the decision-maker requires some additional expertise that may be provided by participants of a human-machine collective intelligence environment. Bearing in mind, 
that using collective expertise is usually rather expensive and can be justified only for important problems, the decision-maker is usually a middle-to-top level manager in terms of typical business hierarchy. After the decision-maker posts the problem to the collective intelligence, he/she may oversee the process of solution and guide it in some way.

Experts possess problem-specific knowledge and may contribute into decision support process in several ways. First, they can propose procedures of obtaining relevant judgements, constructing in an ad hoc way elements of the whole workflow. This can be done not only in a direct manner, but also indirectly, by posting various incentives for other participants. Second, they can use their expertise by providing data as well as processing it to come to some problem-related conclusions. In general, an expert can be anyone - within or without the organization boundary, the difference is mostly in the incentives important for the particular expert.

Service providers design and maintain various software tools, services and datasets that can be used for decision support. Their goal is to receive remuneration for the use of these tools, that is why they are interested in making these services available for other participants of the environment.

The rest of the section introduces foundational technologies and enablers for the proposed environment.

Meeting Collective Intelligence and Artificial Intelligence. Methods of collective intelligence (construed as methods for making people to work together to solve problems) and methods of artificial intelligence are two complementary (in some industries even competing) methods of decision support. Mostly, these approaches are considered as alternative (some tasks due to their nature turn out to be more "convenient" for artificial intelligence methods, and others - for collective), however, the scientists are currently tending to speak about possibility of their joint usage and the potential that human-machine technologies have (Kamar, 2016; Nushi et al., 2017; Verhulst, 2018).

In the proposed environment artificial and collective intelligence are meeting in the following way. The environment itself provides possibility of communication and coordination of agents while working on solving the problem (collective part). Software services have to "understand" common goal and build their strategy (AI part). Besides, some agents can provide application level AI methods.

There are four types of intelligent software services that take part in the functioning of the environment (Figure 1):

- Solver. A software code that can transform a task description in some way, enriching it with some derived knowledge.

- Data/knowledge provider. Interface-wise similar to the previous type, however, only provides some problem-specific information.

- Tool handler. A utility agent that manages human access to some software tools (with GUI). In many cases, certain data processing routines required for decision-making can be implemented with some software (or, SaaS). It is not practical to re-implement it in a new way, however, granting an access to such tools might be useful for all the involved parties.

- Representative. Allowing expert to communicate with other services.

Self-organization protocols taking into account both human and machine agents. One of the distinguishing features of the proposed approach is to overcome the preprogrammed workflows that rigidly govern interaction of participants during decision support and to allow the participants (human and machine agents) to dynamically decide on the details of the workflow unleashing creative potential of humans. Therefore, agents should be able to coordinate and decide on task distribution, roles etc., in other words a group of agents should be able to self-organize.

The protocols of self-organization in such environment have to respect both machine and human requirements. The latter means that widely used models of bio-inspired self-organization turn out to have less potential to be applied, as they are taken mostly from the analysis of primitive behaviors (e.g., of insects). On the other hand, market (or, economics) based models best of all match the assumed business model (on demand service provisioning). Another possible source are socio-inspired mechanisms and protocols which are totally natural for people, and there already exist some attempts to adapt them for artificial systems (Smirnov \& Shilov, 2015). 


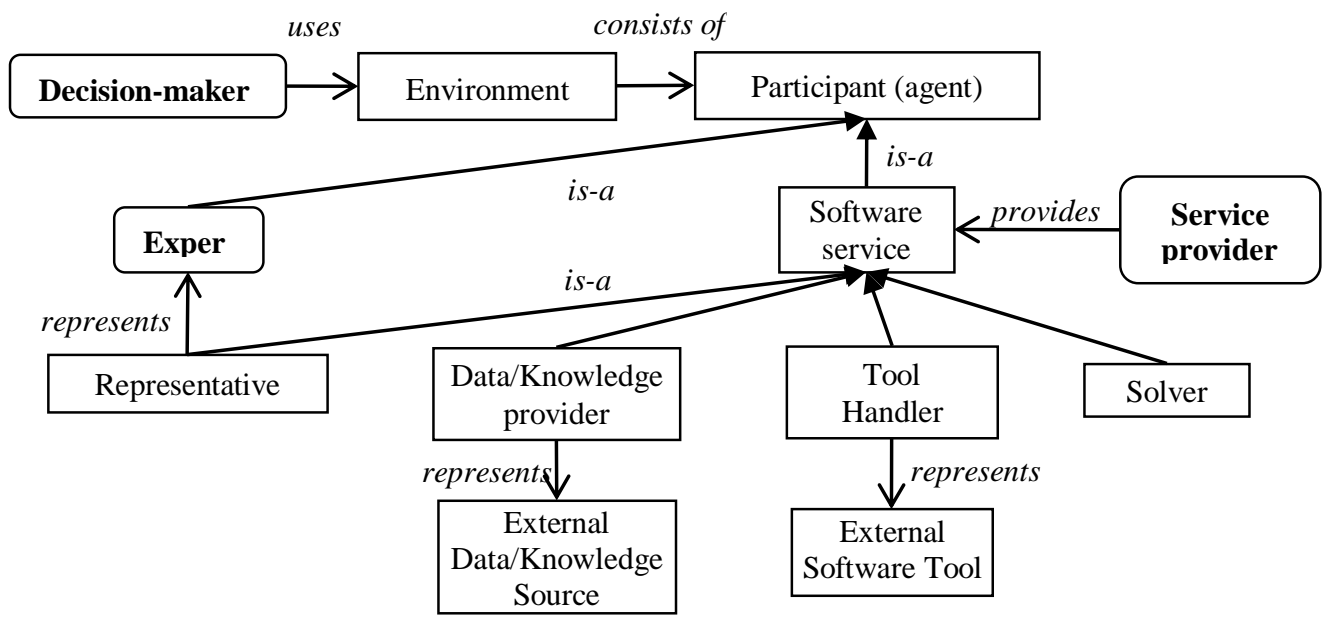

Figure 1. Main entities of the environment and their relationships

Interoperability of agents. To sustain various coordination processes, as well as information flow during decision-making multilevel interoperability has to be provided inside the collaborative environment. This is especially acute in the case of mixed collectives, consisting of human and machine agents.

To implement any self-organization protocols, the participants of the system have to exchange several types of knowledge:

- Domain knowledge. What object and what relationships between objects are in the problem area.

- Task knowledge. Both goal description, and possible conceptualization of the active decision support task, e.g., mapping some concepts to alternatives, functions to criteria.

- Protocol knowledge. Terms of interaction, incentives, roles etc.

It is proposed to use ontologies as the main means ensuring the interoperability. The key role of the ontology model is in its ability to support semantic interoperability as the information represented by ontology can be interpreted both by humans and machines. Potentially, ontology-based information representation can provide the interoperability for all kinds of possible interactions (human-human, human-machine, machine-human). Taking into account the heterogeneity of the participants of the human-machine collective intelligence systems and the multidimensionality of the decision support activities, it is proposed to use multi-aspect ontologies. The multi-aspect ontologies will avoid the need for standardization of all services of environment through providing one aspect (some viewpoint on the domain) to services of one collective (services of one producer, services that jointly solve a certain task, etc.) for the service collaboration.

Soft guidance in collective action. Though the execution process in the proposed environment is self-orchestrated and driven by negotiation protocols, human participants, however, will need intelligent assistance when communicating with other agents in the environment. The role of this assistance is to offer viable organization structures and incentive mechanisms based on current goals. An important aspect during the soft guidance is mapping actions defined by decision-making methodologies to human-computer collaboration scenarios. It means that the environment (or representative service) uses the existing knowledge on decision making process to offer agents viable collaboration structures.

\section{CONCLUSION}

The paper describes a general vision of a novel class of decision support systems. The core of a system (of this novel class) is an environment, providing human-machine collective intelligence. The distinctive features of the proposed environment are: a) support for human and software participants who can build coalitions in order to solve problems and collectively decide on the required workflow, b) support for natural self-organization processes in the community of participants. 
Main foundational technologies and enablers for such environment are: combination of artificial and collective intelligence (in the form of intelligent agents working towards common goal), self-organization protocols supporting both humans and software services, interoperability of agents achieved through the use of multi-aspect ontologies, and soft guidance of collective action by offering viable collaboration structures.

Potential applications of such decision support environment are ubiquitous and influence virtually all areas of human activities, especially in complex domains: smart city, business management, environment problems, government and so on.

\section{ACKNOWLEDGEMENT}

The research is funded by the Russian Science Foundation (project \# 19-11-00126).

\section{REFERENCES}

Ahmad, S., Battle, A., Malkani, Z. and Kamvar, S., 2011. The jabberwocky programming environment for structured social computing. In Proc. 24th Annual ACM Symposium on User Interface Software and Technology, ser. UIST '11, pp. 53-64. ACM.

Barowy, D.W., Curtsinger, C., Berger, E.D. and McGregor, A., 2012. Automan: A platform for integrating human-based and digital computation. SIGPLAN Not., vol. 47, no. 10, pp. 639-654.

Berry, G., 1992. The chemical abstract machine. Theoretical Computer Science, vol. 96, issue 1, pp. 217-248.

Dale, R., Fusaroli, R., Duran, N.D. and Richardson, D.C., 2013. The Self-Organization of Human Interaction. Psychology of Learning and Motivation, vol. 59, pp. 43-95.

Dustdar, S., Nastic, S. and Scekic, O., 2017. Smart Cities: The Internet of Things, People and Systems. Springer.

Forsyth, D.R., 2006. Decision making. In Forsyth, D. R. Group Dynamics (5th Ed.), pp. 317-349. Belmont: CA, Wadsworth, Cengage Learning.

Gorodetskii V.I., 2012. Self-Organization and Multiagent Systems: I. Models of Multiagent Self-Organization. Journal of Computer and Systems Sciences International, vol. 51, no. 2, pp. 256-281.

Kamar, E., 2016. Directions in Hybrid Intelligence: Complementing AI Systems with Human Intelligence. IJCAI Invited Talk: Early Career Spotlight Track.

Kogan, M., 2016. Digital Traces of Online Self-Organizing and Problem Solving in Disaster. In Proceedings of the 19th International Conference on Supporting Group Work - GROUP '16, pp. 479-483. USA: ACM Press.

Kulkarni, A., Can, M. and Hartmann, B., 2012. Collaboratively crowdsourcing workflows with turkomatic. Proceedings of the ACM 2012 Conference on Computer Supported Cooperative Work, Seattle, Washington, USA.

Kulkarni, A.P., Can, M. and Hartmann, B., 2011. Turkomatic: Automatic recursive task and workflow design for mechanical turk. In CHI '11 Ext. Abs. on Human Factors in Comp. Sys., ser. CHI EA '11, pp. 2053-2058. ACM.

Minder, P. and Bernstein, A., 2012. Crowdlang: A programming language for the systematic exploration of human computation systems. In Social Informatics, ser. LNCS, K. Aberer, A. Flache, W. Jager, L. Liu, J. Tang, and C. Guret, Eds. Springer, vol. 7710, pp. 124-137.

Nushi, B., Kamar, E., Horvitz, E. and Kossmann, D., 2017. On human intellect and machine failures: Troubleshooting integrative machine learning systems. 31st AAAI Conference on Artificial Intelligence, pp. 1017-1025.

Retelny, D., Bernstein, M.S. and Valentine, M.A., 2017. No Workflow Can Ever Be Enough: How Crowdsourcing Workflows Constrain Complex Work. In Proc. ACM Human-Computer Interact. 2017. T. 1. № 2. C. Article 89.

Smirnov, A. and Shilov, N., 2015. Service-Based Socio-Cyberphysical Network Modeling for Guided Self-Organization. Procedia Computer Science, vol. 64, pp. 290-297.

Tranquillini, S., Daniel, F., Kucherbaev, P. and Casati, F., 2015. Modeling, enacting, and integrating custom crowdsourcing processes. ACM Trans. Web, vol. 9, no. 2, pp. 7:1-7:43.

Valentine, M.A. et al., 2017. Flash Organizations. In 2017 CHI Conference on Human Factors in Computing Systems - CHI '17, pp. 3523-3537. New York, New York, USA: ACM Press.

Verhulst, S.G., 2018. AI \& Society. vol. 33, issue 2, pp. 293-297.

Viroli, M., Audrito, G., Beal, J., Damiani F. and Pianini D., 2018. Engineering Resilient Collective Adaptive Systems by Self-Stabilisation. Journal ACM Transactions on Modeling and Computer Simulation, Vol. 28, issue 2, article 16. 